\title{
Influence of Breed, Sex and Anatomical Location on Lipid and Fatty Acid Com- position of Bovine Body-cavity Fat
}

\author{
Toyonobu Yoshimura and Kiyoshi Namikawa \\ Department of Animal Science, College of Agriculture, \\ Kyoto University, Kyoto-shi 606
}

(Received July 13, 1984)

\begin{abstract}
In order to examine the effects of breed, sex and anatomical location on the lipid and fatty acid composition of bovine body-cavity fat at a constant weight, bodycavity fat samples were obtained from 5 anatomical locations of the carcasses including Japanese Black, Japanese Black $\times$ Holstein $\left(F_{1}\right)$ and Holstein. The shoulder, loin, ru$\mathrm{mp}$, brisket and flank comprised the locations. The total lipid content was significantly affected by location, being greater in the rump than other locations. As to the lipid composition, only PL class was significantly affected by location, and the percentage of PL was higher in the shoulder than other locations. The total lipid content and lipid composition of bovine body-cavity fat were not so clearly modified by the factors considered in our present study. The fatty acid compositions of TG, FFA and PL classes, however, were significantly affected by breed, sex and/or location showing similar patterns among lipid classes, although some exceptions were observed. In the fatty acid composition of bovine body-cavity fat, the differences among breeds and between sexes showed similar tendencies to those of subcutaneous and intermuscular fats. Namely, Japanese Black were higher in the percentages of C 18:1 and TUSF, and C 18/C 16 ratio than Holstein, and the reverse tendencies were true for the saturated fatty acids, such as $\mathrm{C} 14: 0, \mathrm{C} 16: 0$ and $\mathrm{C} 18: 0$ acids, while $F_{1}$ animals had intermediate values for these fatty acids. Steers showed a tendency to be less abundant in the unsaturated fatty acids than heifers. The differences among locations, however, were smallet than in those of intermuscular fat and then were also not conformable to those of subcutaneous fat.

Jpn. J. Zootech. Sci., 56 (2): 130-136, 1985
\end{abstract}

In our preceding reports concerned with bovine subcutaneous and intermuscular fats $^{1.2}$, the constitutional fatty acids of three major lipid classes were affected by breed, sex and/or anatomical location, and the differences among breeds and between sexes observed in subcutaneous fat were similar to those observed in intermuscular fat, nevertheless locational differences were larger in intermuscular than in subcutaneous fat. These results may suggest that throughout the various bovine adipose tissues, the fatty acid composition is originally governed by genetic and/or physiological conditions which are implicated by breed and sex effects. In order to confirm our hypothesis, it is necessary to get further information about the lipid and fatty acid composition of bovine adipose tissue.

In this study, the effects of breed, sex and anatomical location on the lipid and fatty acid composition of bovine body-cavity fat were investigated at a constant slaughter weight. 


\section{Materials and Methods}

The experimental animals consisted of Japanese Black ( 9 steers), Holstein ( 8 steers and 2 heifers) and Japanese Black $\times$ Holstein $\left(F_{1}\right)$ (6 steers and 6 heifers), as described previously ${ }^{1)}$. Body-cavity fat samples were taken from five locations on the left side of each carcass, including the shoulder (body-cavity side at the 7-8 th thoracic vertebra), loin (the periphery of the psoas major muscle), rump (pelvic cavity close to the obturator internus et externus muscle), brisket (over the transversus thoracis muscle) and flank (the end of the rectus abdominus muscle).

Total lipid content, lipid composition, and fatty acid composition of triglycerides (TG), free fatty acids (FFA) and phospholipids (PL) fraction were determined according to the analytical procedure outlined previously").

Data were analyzed by least-squares analysis of variance according to the procedure by HARVEY ${ }^{3)}$, using the computer program, LSML 76 $6^{4}$. The mathematical model involved initially four fixed effects (i. e., breed, sex, anatomical location and nutritional level); the two way interaction between breed and location; and pooled and withinbreed partial regression on slaughter weight (average $523 \mathrm{~kg}$ ). DuncaN's new multiple range test ${ }^{5)}$ with KRAMER's modification ${ }^{61}$ was used to determine the significance of differences among means.

\section{Results and Discussion}

The lipid and fatty acid profiles of body-cavity fat were similar to those of subcutaneous $^{1)}$ and of intermuscular fats ${ }^{2}$. Therefore, the lipid and fatty acid compositions were quantified and statistically analyzed in the same manner described previously ${ }^{1}$. In the results of analysis of variance, the breed $\times$ location interaction, how ever, was not significant. Therefore, the results derived from the analysis of variance according to the model without the interaction were shown in the following tables.

Total Lipid Content and Lipid Composition

The least-squares means for the total lipid content and lipid composition by breed, sex and location are given in Table 1 . In body-cavity fat, as a whole, the total lipid content and percentage of TG were higher, and the percentages of PL and FFA were lower than in subcutaneous ${ }^{1)}$ and intermuscular fats ${ }^{2}$. In the results of analyses of variance, the total lipid content and the percentage of PL were significantly affected only by location. The rump fat had a greater total lipid content than the fats from other locations. The percentage of PL was higher in the shoulder than in any other locations. None of the total lipid content and lipid composition were significantly affected by breed and sex. Thus, the total lipid content and lipid composition were less affected by the factors considered in this study. These tendencies are identical with the results of subcutaneous fat ${ }^{1 /}$ but differ from those of intermuscular fat $^{2)}$.

Fatty Acid Composition of TG, FFA and $P L$

In body-cavity fat, as a whole, the percentages of $C 18: 1$ and $C 16: 1$ of any 
Table 1. Least-squares means and standard errors for total lipid content and lipid composition ${ }^{1,21}$

\begin{tabular}{|c|c|c|c|c|}
\hline \multirow{2}{*}{ Effect } & \multirow{2}{*}{$\begin{array}{c}\text { Total lipids } \\
\text { (mg/g wet tissue) }\end{array}$} & \multicolumn{3}{|c|}{ Lipid class } \\
\hline & & $\begin{array}{l}\text { TG } \\
(\%)\end{array}$ & $\begin{array}{l}\text { PL } \\
(\%)\end{array}$ & $\begin{array}{l}\text { FFA }^{3 \mid} \\
(\%)\end{array}$ \\
\hline \multicolumn{5}{|l|}{ Breed } \\
\hline Japanese Black & $926.8(8.1)$ & $97.7(0.2)$ & $0.6(0.1)$ & $0.9(0.1)$ \\
\hline $\mathrm{B} \times \mathrm{H} \quad\left(\mathrm{F}_{1}\right)$ & $924.7(7.0)$ & $97.5(0.2)$ & $0.7(0.1)$ & $0.9(0.1)$ \\
\hline Holstein & $912.1(6.5)$ & $97.9(0.2)$ & $0.5(0.1)$ & $0.7(0.1)$ \\
\hline \multicolumn{5}{|l|}{ Sex } \\
\hline Steer & $921.8(5.7)$ & $97.6(0.1)$ & $0.5(0.1)$ & $0.8(0.05)$ \\
\hline Heifer & $920.5(8.5)$ & $97.7(0.2)$ & $0.6(0.1)$ & $0.8(0.1)$ \\
\hline Location & $* *$ & & $* *$ & \\
\hline Shoulder & $911.5(7.3)^{b}$ & $97.4(0.2)$ & $0.8(0.1)^{a}$ & $0.8(0.1)$ \\
\hline Loin & $917.3^{\mathrm{b}}$ & 97.7 & $0.6^{\mathrm{b}}$ & 0.8 \\
\hline Rump & $938.8^{\mathrm{a}}$ & 97.9 & $0.4^{\mathrm{b}}$ & 0.8 \\
\hline Brisket & $920.6^{b}$ & 97.7 & $0.5^{b}$ & 0.8 \\
\hline Flank & $917.8^{b}$ & 97.6 & $0.5^{\mathrm{b}}$ & 0.9 \\
\hline
\end{tabular}

1) The relative amounts of lipid classes are expressed as relative weight percentages of TG, PL, FFA, CH and DG. 2) The figures within parentheses show the standard errors. 3) The abbreviation used are as follows: TG, triglycerides; PL, phospholipids; FFA, free fatty acids. $* \mathrm{P}<.05 . * * \mathrm{P}<.01 \quad \mathrm{a}, \mathrm{b}$ Within a main effect, means in the same column with no common superscripts differ significantly $(P<.05)$.

lipid classes were lower by ca. 2.0 and $1.0 \%$, respectively, while those of C $18: 0$ were higher by $3-5 \%$ than in intermuscular fat ${ }^{2)}$ (Tables 2,3 and 4 ). These percentages were fairly consistent with those of bovine visceral fat (YoshImURA and NAMIKAWA, unpublished). Although little information was available as to the fatty acid composition of bovine body-cavity fat, HOOD and THORTON ${ }^{7}$ reported that bovine channel fat, which is identical with the rump region in our study, had a similar fatty acid composition to perinephric fat.

In the TG class, each fatty acid, total unsaturated fatty acids (TUSF) and (C 18: $0+\mathrm{C} 18: 1) /(\mathrm{C} 16: 0+\mathrm{C} 16: 1)$ ratio (C 18/C 16 ratio) were affected by breed, sex and/or location (Table 2). The breed difference of compositional fatty acids were most remarkable in $\mathrm{C} 18: 1$ acid which was most aboundantly contained in bovine body-cavity fat $(\mathrm{P}<.01)$. Japanese Black had the highest percentage of $\mathrm{C} 18: 1$ $(49.3 \%)$, while Holstein had the lowest one $(40.9 \%)$ among the tested breeds. For the saturated fatty acids, such as $\mathrm{C} 14: 0, \mathrm{C} 16: 0$ and $\mathrm{C} 18: 0$ acids, the reverse tendencies were true. The remarkable difference in $\mathrm{C} 18: 1$ acid brought about the highest percentage of TUSF and the highest value of $\mathrm{C} 18 / \mathrm{C} 16$ ratio in Japanese Black. For these fatty acids and indices, $F_{1}$ animals had a tendency to have intermediate values between their parental breeds, excluding $\mathrm{C} 18: 2$ which was most abundant in $F_{1}$ animals, and the difference between $F_{1}$ animals and Holstein was not 
Table 2. Least-squares means and standard errors for the fatty acid composition of triglycerides $^{1,2)}$

\begin{tabular}{|c|c|c|c|c|c|c|c|c|}
\hline \multirow[b]{2}{*}{ Effect } & \multicolumn{8}{|c|}{ Fatty acid $^{8)}$} \\
\hline & $\begin{array}{c}\text { C 14:0 } \\
(\%)\end{array}$ & $\begin{array}{c}\mathrm{C} 16: 0 \\
(\%)\end{array}$ & $\begin{array}{c}\text { C 16:1 } \\
(\%)\end{array}$ & $\begin{array}{c}\mathrm{C} 18: 0 \\
(\%)\end{array}$ & $\begin{array}{c}\text { C 18:1 } \\
(\%)\end{array}$ & $\begin{array}{c}\text { C } 18: 2 \\
(\%)\end{array}$ & $\begin{array}{c}\left.\mathrm{TUSF}^{4}\right) \\
(\%)\end{array}$ & $\mathrm{C} 18 / \mathrm{C} 16^{5}$ \\
\hline Breed & $* *$ & $* *$ & & $* *$ & $* *$ & $* *$ & $* *$ & $* *$ \\
\hline Japanese Black & $\begin{array}{l}2.3^{b} \\
(0.1)\end{array}$ & $\begin{array}{c}22.5^{b} \\
(0.5)\end{array}$ & $\begin{array}{c}4.1 \\
(0.2)\end{array}$ & $\begin{array}{l}16.2^{b} \\
(0.8)\end{array}$ & $\begin{array}{l}49.3^{a} \\
(0.8)\end{array}$ & $\begin{array}{l}1.4^{b} \\
(0.2)\end{array}$ & $\begin{array}{l}56.0^{\mathrm{a}} \\
(1.0)\end{array}$ & $\begin{array}{c}2.5^{\mathrm{a}} \\
(0.1)\end{array}$ \\
\hline $\mathrm{B} \times \mathrm{H} \quad\left(\mathrm{F}_{1}\right)$ & $\begin{array}{r}2.4^{\mathrm{b}} \\
(0.1)\end{array}$ & $\begin{array}{l}23.8^{\mathrm{a}} \\
(0.4)\end{array}$ & $\begin{array}{c}3.9 \\
(0.1)\end{array}$ & $\begin{array}{l}16.9^{b} \\
(0.7)\end{array}$ & $\begin{array}{l}45.7^{\mathrm{b}} \\
(0.7)\end{array}$ & $\begin{array}{c}3.1^{\mathrm{a}} \\
(0.2)\end{array}$ & $\begin{array}{l}53.8^{b} \\
(0.8)\end{array}$ & $\begin{array}{c}2.3^{b} \\
(0.1)\end{array}$ \\
\hline Holstein & $\begin{array}{c}2.9^{\mathrm{a}} \\
(0.1)\end{array}$ & $\begin{array}{l}24.7^{4} \\
(0.4)\end{array}$ & $\begin{array}{c}3.7 \\
(0.1)\end{array}$ & $\begin{array}{l}20.5^{a} \\
(0.6)\end{array}$ & $\begin{array}{l}40.9^{c} \\
(0.7)\end{array}$ & $\begin{array}{c}3.0^{a} \\
(0.2)\end{array}$ & $\begin{array}{l}48.7^{c} \\
(0.8)\end{array}$ & $\begin{array}{c}2.2^{b} \\
(0.1)\end{array}$ \\
\hline Sex & *** & & & & ** & $*$ & $*$ & \\
\hline Steer & $\begin{array}{r}2.8^{\mathrm{a}} \\
(0.1)\end{array}$ & $\begin{array}{l}23.9 \\
(0.4)\end{array}$ & $\begin{array}{l}3.7 \\
(0.1)\end{array}$ & $\begin{array}{l}18.5 \\
(0.5)\end{array}$ & $\begin{array}{l}44.0^{b} \\
(0.6)\end{array}$ & $\begin{array}{c}2.7^{\mathrm{a}} \\
(0.1)\end{array}$ & $\begin{array}{l}51.7^{\mathrm{a}} \\
(0.7)\end{array}$ & $\begin{array}{l}2.3 \\
(0.05)\end{array}$ \\
\hline Heifer & $\begin{array}{r}2.3^{b} \\
(0.1)\end{array}$ & $\begin{array}{l}23.2 \\
(0.6)\end{array}$ & $\begin{array}{c}4.0 \\
(0.2)\end{array}$ & $\begin{array}{l}17.2 \\
(0.8)\end{array}$ & $\begin{array}{r}46.5^{a} \\
(0.9)\end{array}$ & $\begin{array}{l}2.2^{b} \\
(0.2)\end{array}$ & $\begin{array}{l}54.0^{a} \\
(1.0)\end{array}$ & $\begin{array}{c}2.4 \\
(0.1)\end{array}$ \\
\hline Location & $* *$ & & $* *$ & $* *$ & $* *$ & $* *$ & $* *$ & $* *$ \\
\hline Shoulder & $\begin{array}{c}2.5^{\mathrm{b}} \\
(0.1)\end{array}$ & $\begin{array}{l}23.4 \\
(0.5)\end{array}$ & $\begin{array}{c}3.6^{b} \\
(0.1)\end{array}$ & $\begin{array}{l}20.2^{a} \\
(0.7)\end{array}$ & $\begin{array}{l}43.2^{b} \\
(0.8)\end{array}$ & $\begin{array}{c}2.8^{4} \\
(0.2)\end{array}$ & $\begin{array}{c}50.8^{b} \\
(0.9)\end{array}$ & $\begin{array}{c}2.4^{\mathbf{a}} \\
(0.1)\end{array}$ \\
\hline Loin & $2.5^{b}$ & 22.9 & $3.8^{\mathrm{b}}$ & $18.2^{b}$ & $45.8^{a}$ & $2.4^{\mathrm{ab}}$ & $53.3^{\mathrm{a}}$ & 2. $4^{\mathrm{a}}$ \\
\hline Rump & $2.6^{\mathrm{b}}$ & 23.7 & $3.8^{b}$ & $18.0^{\circ}$ & $45.0^{a}$ & $2.6^{\mathrm{a}}$ & $52.4^{\mathrm{ab}}$ & $2.3^{\mathrm{ab}}$ \\
\hline Brisket & $2.5^{b}$ & 23.4 & $3.9^{\mathrm{b}}$ & $17 \cdot 3^{b}$ & $45.9^{a}$ & $2.5^{\mathrm{a}}$ & $53.5^{a}$ & 2. $3^{a}$ \\
\hline Flank & $2.8^{\mathrm{a}}$ & 24.3 & 4. $2^{\mathrm{a}}$ & $15.6^{\circ}$ & $46.5^{a}$ & 2. $1^{\mathrm{b}}$ & $54.1^{*}$ & $2.2^{b}$ \\
\hline
\end{tabular}

1) Fatty acids are expressed as the relative weight percent of 12 fatty acid methyl esters. 2) The figures within parentheses show the standard errors. 3) Number of carbon atoms: number of double bonds. 4) Total unsaturated fatty acids. 5) (C18:0+C18:1)/(C16:0+ C16:1) ratio. * $\mathrm{P}<.05, * * \mathrm{P}<.01 \cdot \mathrm{a}, \mathrm{b}, \mathrm{c}$ Within a main effect, means in the same column with no common superscripts differ significantly $(\mathrm{P}<.05)$.

significant. Significant differences between sexes were found for C $14: 0, \quad$ C $18: 1$, C $18: 2$ and TUSF. Steers had a smaller portion of $C 18: 1$ acid than heifers. The differences in $\mathrm{C} 18: 1$ acid of the body-cavity fat were comparable with that of subcutaneous $^{1)}$ and intermuscular fats ${ }^{2)}$. Significant locational differences were observed in each fatty acids and indices, except for $\mathrm{C} 16: 0$ acid. In most of these cases, only one location was different from others. Therefore, the fatty acid composition of the body-cavity fat was not so clearly affected by the location, as well as that of subcutaneous fat ${ }^{11}$.

The least-squares means for the fatty acid composition of FFA and PL classes are shown in Tables 3 and 4, respectively. Similar breed and locational differences, which were observed in TG class, were also detected in both of FFA and PL classes. Arachidonic acid (C $20: 4$ ) of PL class were significantly affected by breed and location. Among breeds, Japanese Black had the lowest percentage. Same species of fatty acids existing at different lipid classes were similarly affected by the factors considered in present study, in agreement with our preceding reports ${ }^{1,2}$.

As described in our foregoing results ${ }^{1,2}$, the constitutional fatty acids of each 
Table 3. Least-squares means and standard errors for the fatty acid composition of free fatty acids ${ }^{1,2)}$

\begin{tabular}{|c|c|c|c|c|c|c|c|c|}
\hline \multirow[b]{2}{*}{ Effect } & \multicolumn{8}{|c|}{ Fatty acid ${ }^{3)}$} \\
\hline & $\begin{array}{c}\mathrm{C} 14: 0 \\
(\%)\end{array}$ & $\begin{array}{c}\text { C 16:0 } \\
(\%)\end{array}$ & $\begin{array}{c}\mathrm{C} 16: 1 \\
(\%)\end{array}$ & $\begin{array}{c}\text { C 18:0 } \\
(\%)\end{array}$ & $\underset{(\%)}{C} 18: 1$ & $\underset{(\%)}{C 18: 2}$ & $\underset{(\%)}{\text { TUSF }}$ & $\left.\mathrm{C} 18 / \mathrm{C} 16^{5}\right)$ \\
\hline Breed & & $* *$ & & $* *$ & $* *$ & $* *$ & $* *$ & $* *$ \\
\hline Japanese Black & $\begin{array}{c}1.9 \\
(0.1)\end{array}$ & $\begin{array}{l}16.4^{b} \\
(0.8)\end{array}$ & $\begin{array}{c}5.5 \\
(0.3)\end{array}$ & $\begin{array}{l}11.9^{6} \\
(0.9)\end{array}$ & $\begin{array}{l}57.1^{a} \\
(1.3)\end{array}$ & $\begin{array}{c}2.1^{0} \\
(0.3)\end{array}$ & $\begin{array}{l}66.3^{a} \\
(1.5)\end{array}$ & $\begin{array}{c}3.2^{\mathbf{a}} \\
(0.1)\end{array}$ \\
\hline $\mathrm{B} \times \mathrm{H} \quad\left(\mathrm{F}_{1}\right)$ & $\begin{array}{c}2.0 \\
(0.1)\end{array}$ & $\begin{array}{l}17.7^{a} \\
(0.6)\end{array}$ & $\begin{array}{c}5.4 \\
(0.2)\end{array}$ & $\begin{array}{l}12.4^{b} \\
(0.8)\end{array}$ & $\begin{array}{l}53.5^{\mathrm{b}} \\
(1.1)\end{array}$ & $\begin{array}{c}4.1^{\mathrm{a}} \\
(0.3)\end{array}$ & $\begin{array}{l}64.5^{b} \\
(1.3)\end{array}$ & $\begin{array}{c}2.9^{b} \\
(0.1)\end{array}$ \\
\hline Holstein & $\begin{array}{c}2.2 \\
(0.1)\end{array}$ & $\begin{array}{r}19.2^{a} \\
(0.6)\end{array}$ & $\begin{array}{c}4.9 \\
(0.2)\end{array}$ & $\begin{array}{r}15.9^{3} \\
(0.7)\end{array}$ & $\begin{array}{r}48.5^{c} \\
(1.1)\end{array}$ & $\begin{array}{l}4.3^{2} \\
(0.3)\end{array}$ & $\begin{array}{l}59.0^{6} \\
(1.2)\end{array}$ & $\begin{array}{c}2.7^{b} \\
(0.1)\end{array}$ \\
\hline Sex & & & & & & $*$ & & \\
\hline Steer & $\begin{array}{l}2.1 \\
(0.1)\end{array}$ & $\begin{array}{l}17.3 \\
(0.5)\end{array}$ & $\begin{array}{c}5.2 \\
(0.2)\end{array}$ & $\begin{array}{l}13.3 \\
(0.6)\end{array}$ & $\begin{array}{l}53.2 \\
(0.9)\end{array}$ & $\begin{array}{l}4.1^{2} \\
(0.2)\end{array}$ & $\begin{array}{l}64.0 \\
(1.1)\end{array}$ & $\begin{array}{l}3.0 \\
(0.1)\end{array}$ \\
\hline Heifer & $\begin{array}{l}1.9 \\
(0.1)\end{array}$ & $\begin{array}{l}18.3 \\
(0.8)\end{array}$ & $\begin{array}{c}5.3 \\
(0.3)\end{array}$ & $\begin{array}{l}13.6 \\
(1.0)\end{array}$ & $\begin{array}{l}52.9 \\
(1.4)\end{array}$ & $\begin{array}{c}2.9^{b} \\
(0.4)\end{array}$ & $\begin{array}{l}62.5 \\
(1.6)\end{array}$ & $\begin{array}{c}2.9 \\
(0.1)\end{array}$ \\
\hline Location & & & $* *$ & $* *$ & $*$ & & $* * *$ & \\
\hline Shoulder & $\begin{array}{l}1.9 \\
(0.1)\end{array}$ & $\begin{array}{l}18.1 \\
(0.7)\end{array}$ & $\begin{array}{l}5.0^{b} \\
(0.3)\end{array}$ & $\begin{array}{l}15.0^{a} \\
(0.8)\end{array}$ & $\begin{array}{l}51.3^{b} \\
(1.2)\end{array}$ & $\begin{array}{c}3.6 \\
(0.3)\end{array}$ & $\begin{array}{l}61.2^{c} \\
(1.4)\end{array}$ & $\begin{array}{c}2.9 \\
(0.1)\end{array}$ \\
\hline Loin & 1.9 & 17.8 & $4.9^{b}$ & $14.8^{3}$ & $52.2^{b}$ & 3.5 & $62.0^{b c}$ & 3.0 \\
\hline Rump & 2.1 & 18.0 & $5.0^{6}$ & $13.2^{b}$ & $52.9^{\mathrm{ab}}$ & 3.7 & $63.0^{a b c}$ & 2.9 \\
\hline Brisket & 2.0 & 17.7 & $5.4^{a b}$ & 12. $7^{b}$ & $53.6^{a b}$ & 3.6 & $64.2^{a b}$ & 2.9 \\
\hline Flank & 2.1 & 17.2 & $5.8^{a}$ & $11.5^{b}$ & $55.2^{\circ}$ & 3.2 & $65.8^{\text {a }}$ & 2.9 \\
\hline
\end{tabular}

1) Fatty acids are expressed as the relative weight percent of 12 fatty acid methyl esters. 2) The figures within parentheses show the standard errors. 3) Number of carbon atoms: number of double bonds. 4) Total unsaturated fatty acids. 5) (C18:0+C18:1)/(C16:0+ C16:1) ratio. $* \mathrm{P}<.05, * * \mathrm{P}<.01$. $\mathrm{a}, \mathrm{b}, \mathrm{c}$ Within a main effect, means in the same column with no common superscripts differ significantly $(\mathrm{P}<.05)$.

lipid class were made up of primary lipids which varied with breed, sex and location. Particularly the breed differences were remarkable and furthermore, the interaction between breed and location was negligible in any of fatty acids. These findings suggest that the similar breed difference in the fatty acid composition were entirely observed throughout all locations of the body-cavity fat. In addition, the differences among breeds and between sexes were similar to those of subcutaneous and intermuscular fats ${ }^{1.2}$, although the mean percentages of each fatty acid were different among adipose tissues, suggesting that the influences of breed and sex were common phenomena in any body fat depots. On the other hand, the locational differences in the fatty acid composition of the body-cavity fat were smaller than those of the intermuscular fat $^{21}$ and then were not comformable to those of the subcutaneous fat ${ }^{11}$. That is to say, it may be expected that there is some interaction between depot and anatomical location on bovine fatty acid composition. The integrated search for the clarification of the properties of the variation detected in the fatty acid composition of bovine adipose tissue may be required. 
Table 4. Least-squares means and standard errors for the fatty acid composition of phospholipids ${ }^{1,2)}$

\begin{tabular}{|c|c|c|c|c|c|c|c|c|c|}
\hline \multirow{2}{*}{ Effect } & \multicolumn{9}{|c|}{ Fatty acids ${ }^{s !}$} \\
\hline & $\begin{array}{c}C 14: 0 \\
(\%)\end{array}$ & $\begin{array}{c}C 16: 0 \\
(\%)\end{array}$ & $\begin{array}{c}\text { C16:1 } \\
(\%)\end{array}$ & $\begin{array}{c}\text { C18:0 } \\
(\%)\end{array}$ & $\begin{array}{c}C 18: 1 \\
(\%)\end{array}$ & $\begin{array}{c}\mathrm{C} 18: 2 \\
(\%)\end{array}$ & $\begin{array}{c}\mathrm{C} 20: 4 \\
(\%)\end{array}$ & $\begin{array}{c}\text { TUSF } \\
(\%)\end{array}$ & $\mathrm{C} 18 / \mathrm{C} 16^{5)}$ \\
\hline Breed & & $* *$ & $* *$ & $* *$ & $* *$ & $*$ & $* *$ & $* *$ & $* *$ \\
\hline Japanese Black & $\begin{array}{c}1.4 \\
(0.2)\end{array}$ & $\begin{array}{l}20.3^{b} \\
(0.8)\end{array}$ & $\begin{array}{c}4.1^{a} \\
(0.4)\end{array}$ & $\begin{array}{l}17.5^{a} \\
(1.1)\end{array}$ & $\begin{array}{l}40.7^{3} \\
(1.3)\end{array}$ & $\begin{array}{c}3.6^{b} \\
(0.3)\end{array}$ & $\begin{array}{c}3.4^{\mathrm{b}} \\
(0.4)\end{array}$ & $\begin{array}{l}57.8^{\circ} \\
(1.3)\end{array}$ & $\begin{array}{c}2.4^{a} \\
(0.1)\end{array}$ \\
\hline $\mathrm{B} \times \mathrm{H} \quad\left(\mathrm{F}_{1}\right)$ & $\begin{array}{c}1.6 \\
(0.2)\end{array}$ & $\begin{array}{l}22.2^{\mathrm{a}} \\
(0.7)\end{array}$ & $\begin{array}{c}4.3^{a} \\
(0.3)\end{array}$ & $\begin{array}{l}18.3^{\mathbf{a}} \\
(0.9)\end{array}$ & $\begin{array}{l}36.4^{\mathrm{b}} \\
(1.1)\end{array}$ & $\begin{array}{c}4.2^{\mathrm{a}} \\
(0.3)\end{array}$ & $\begin{array}{c}3.5^{b} \\
(0.4)\end{array}$ & $\begin{array}{c}54.3^{\mathrm{b}} \\
(1.1)\end{array}$ & ${ }^{2.1}{ }^{\mathrm{b}}$ \\
\hline Holstein & $\begin{array}{c}1.5 \\
(0.2)\end{array}$ & $\begin{array}{r}22.5^{a} \\
(0.6)\end{array}$ & $\begin{array}{r}3.2^{b} \\
(0.3)\end{array}$ & $\begin{array}{c}23.1^{b} \\
(0.9)\end{array}$ & $\begin{array}{l}32.1^{\circ} \\
(1.1)\end{array}$ & $\begin{array}{r}4.5^{2} \\
(0.3)\end{array}$ & $\begin{array}{r}4.6^{2} \\
(0.3)\end{array}$ & $\begin{array}{l}49.9^{c} \\
(1.0)\end{array}$ & $\begin{array}{c}2.2^{b} \\
(0.1)\end{array}$ \\
\hline \multicolumn{10}{|l|}{ Sex } \\
\hline Steer & $\begin{array}{c}1.6 \\
(0.1)\end{array}$ & $\begin{array}{l}22.1 \\
(0.5)\end{array}$ & $\begin{array}{c}3.7 \\
(0.3)\end{array}$ & $\begin{array}{c}20.4 \\
(0.8)\end{array}$ & $\begin{array}{l}36.2 \\
(0.9)\end{array}$ & $\begin{array}{c}3.8 \\
(0.2)\end{array}$ & $\begin{array}{c}3.5 \\
(0.3)\end{array}$ & $\begin{array}{l}52.8 \\
(0.9)\end{array}$ & $\begin{array}{c}2.3 \\
(0.1)\end{array}$ \\
\hline Heifer & $\begin{array}{c}1.4 \\
(0.2)\end{array}$ & $\begin{array}{l}21.3 \\
(0.8)\end{array}$ & $\begin{array}{c}4.1 \\
(0.4)\end{array}$ & $\begin{array}{l}18.8 \\
(1.1)\end{array}$ & $\begin{array}{l}36.6 \\
(1.4)\end{array}$ & $\begin{array}{c}4.4 \\
(0.4)\end{array}$ & $\begin{array}{c}4.1 \\
(0.4)\end{array}$ & $\begin{array}{l}55.2 \\
(1.3)\end{array}$ & $\begin{array}{c}2.2 \\
(0.1)\end{array}$ \\
\hline Location & & & $*$ & & & & $* *$ & * & \\
\hline Shoulder & $\begin{array}{l}1.6 \\
(0.2)\end{array}$ & $\begin{array}{l}22.7 \\
(0.7)\end{array}$ & $\begin{array}{l}4.2^{\mathrm{a}} \\
(0.3)\end{array}$ & $\begin{array}{l}20.3 \\
(1.0)\end{array}$ & $\begin{array}{l}34.6 \\
(1.2)\end{array}$ & $\begin{array}{c}3.7 \\
(0.3)\end{array}$ & $\begin{array}{c}2.9^{b} \\
(0.4)\end{array}$ & $\begin{array}{l}51.7^{b} \\
(1.2)^{2}\end{array}$ & $\begin{array}{c}2.1 \\
(0.1)\end{array}$ \\
\hline Loin & 1.5 & 20.8 & $3.9^{\mathrm{ab}}$ & 19.9 & 36.6 & 4.2 & $3.8^{\mathrm{a}}$ & $54.6^{a}$ & 2.3 \\
\hline Rump & 1.4 & 21.7 & $3.3^{b}$ & 20.3 & 36.5 & 4.2 & $4.0^{a}$ & $53.7^{a b}$ & 2.4 \\
\hline Brisket & 1.4 & 21.5 & $3.8^{\mathrm{ab}}$ & 18.9 & 36.9 & 4.4 & $4.2^{a}$ & $55.1^{2}$ & 2.2 \\
\hline Flank & 1.6 & 21.7 & $4.1^{\mathrm{a}}$ & 18.8 & 37.2 & 4.0 & $4.1^{a}$ & $54.8^{\mathrm{a}}$ & 2.2 \\
\hline
\end{tabular}

1) Fatty acids are expressed as the relative weight percent of 15 fatty acid methyl esters. 2) The figures within parentheses show the standard errors. 3) Number of carbon atoms: number of double bonds. 4) Total unsaturated fatty acids, 5) (C18:0+C18:1)/(C16:0+ C16:1) ratio. * $\mathrm{P}<.05$, ** $\mathrm{P}<.01$. a, b, c Within a main effect, means in the same column with no common superscripts differ significantly $(\mathrm{P}<.05)$.

\section{Acknowledgements}

This work was supported in part by two Scientific Research Grants from the Ministry of Education, Science and Culture (Grant No. 086032 and 286057).

We are greatful to Dr. T. Fukushima (Kobe Univ.), Dr. M. Zenbayashi (the Livestock Farm, Kyoto Univ.), Mr. K. Mrtani (the University Farm, Hiroshima Univ.) and their staffs for their cooperation in feeding and management of experimental animals.

\section{References}

1) Yoshimura, T. and K. Namikawa, Jpn. J. Zootech. Sci., 54: 97-105. 1983.

2) Yoshimura, T. and K. Namikawa, Jpn. J. Zootech. Sci., 56: 122-129, 1985.

3) Harvey, W. R., Least-squares Analysis of Data with Unequal Subclass Numbers. A. R. S., 20-8. U. S. D. A. 1960.

4) Harvey, W. R., User's Guide for LSML 76-Mixed Model Least-squares and Maximum Liklihood Computer Program. Mimeo. Ohio State Univ. Columbus. 1977.

5) Duncan, D. B., Biometrics, 11: 1-42. 1955.

6) KRAMER, C. Y., Biometrics, 12: 307-310, 1956.

7) Hood, R. L. and Thornton, Aust. J. Agric. Res., 27: 895-902. 1976. 


\title{
牛の体腔内脂肪中脂質および脂肪酸組成に対する品種, 性および蓄積部位の影響
}

\author{
吉村豊信・並河 澄
}

京都大学農学部, 京都市 606

牛の体腔内脂肪組織中脂質吕よび脂肪酸組成に対する 品種，性扣よび部位の影響を゙、一定屠殺時体重をー゙ース として比校した，材料は，黒毛和種 (含：9頭)，ホルス タイン種 (舌：8頭○：2 頭) 扣上び墨毛和種 $\times$ ホルス

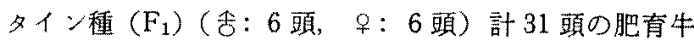
（平均屠殺時体重 $523 \mathrm{~kg}$ ) の枝肉加ら，解体時に得た 5 部位の休腔内脂肪（肩，腰，慇，胸，膁）を用い，総脂 質含量，脂質組成お゙よびトリグリセリド（TG），遊離脂 肪酸 (FFA)，リン脂資 (PL) の各画分の脂肪酸組成を 求めた，総脂筫含量に関しては，部位間の変動にの及有 意性が認められ，蟹部体腔内脂肪で他の部位より高かっ た，脂筫組成に対する各要因の影響は，PL にの灭部位 閒で有意性が認められ，肩部で高かった，以上のように， 体腔内脂肪の空脂質含量や脂質組成は，取り上げた要因 によってそれほど影響されなかった。しかし，主要画分
を構成する脂肪酸は, 多少とも品種, 性および部位の影 學を有意に受ける場合が多く，しかも，その差異は，心 ずれの画分の脂肪酸組成にも共通する㑯向が㤎められ た. また，品種間や性間に認められた体腔内脂肪の脂肪 酸組成の盖は，皮下执よび筋間脂肪のそれに類似した 傾向を示した。すなわち, 体腔内脂肪においても, 品種 間では, 黑毛和種がホルスタイン種よりもC 18:1, TUSF （総不飽和脂肪酸割合）に富み，C 18/C 16 比が高く，飽 和脂肪酸が少ないのに対して， F 1 は両品種の中間值を とる候向を示した。竜た，去勢牛は，末経産牛よりる館 和脂肪酸が多かった，一方，体腔内脂肪における脂肪酸 組成の部位閆の差異は，効閒脂肪の場合よりる小さく， 皮下脂肪の部位間の差異に認められた傾向とる一致しな かった。

日蓄会報，56 (2)：130-136，1985 